Short title: LARGE-SCALE MAGNETIC FIELD GENERATION

\title{
GENERATION OF MULTISCALE MAGNETIC FIELD BY PARITY-INVARIANT TIME-PERIODIC FLOWS
}

\author{
V.A. Zheligovsky ${ }^{a, b, c, 1}$, O.M. Podvigina ${ }^{a, b, c, 2}$ \\ ${ }^{a}$ Observatoire de la Côte d'Azur, CNRS U.M.R. 6529, \\ BP 4229, 06304 Nice Cedex 4, France \\ ${ }^{b}$ International Institute of Earthquake Prediction Theory \\ and Mathematical Geophysics, \\ 79 bldg.2, Warshavskoe ave., 117556 Moscow, Russian Federation \\ ${ }^{c}$ Laboratory of general aerodynamics, Institute of Mechanics, \\ Lomonosov Moscow State University, \\ 1, Michurinsky ave., 119899 Moscow, Russian Federation \\ Submitted to Geophysical and Astrophysical Fluid Dynamics
}

\begin{abstract}
We study generation of magnetic fields involving large spatial scales by time- and space-periodic small-scale parity-invariant flows. The anisotropic magnetic eddy diffusivity tensor is calculated by the standard procedure involving expansion of magnetic modes and their growth rates in power series in the scale ratio. Our simulations, conducted for flows with random harmonic composition and exponentially decaying energy spectra, demonstrate that enlargement of the spatial scale of magnetic field is beneficial for generation by time-periodic flows. However, they turn out, in general, to be less efficient dynamos, than steady flows.
\end{abstract}

Key words. Kinematic magnetic dynamo, slow dynamo, time-periodic flow, asymptotic expansion, Floquet problem, magnetic modes, magnetic eddy diffusivity.

\footnotetext{
${ }^{1}$ E-mail: vlad@mitp.ru

${ }^{2}$ E-mail: olgap@mitp.ru
} 
The present work is an extension of the studies carried out by Lanotte et al. (2000) and Zheligovsky et al. (2001), who found steady parity-invariant flows with a negative magnetic eddy diffusivity to be quite common. A similar investigation for turbulent flows is desirable, especially given that flows in experimental dynamos are necessarily turbulent (see discussion ibid.). Some time-dependent flows are known to be unable to sustain negative magnetic eddy diffusivity - namely, the flows, $\delta$-correlated in time at each point in space. They advect a mean magnetic field as a passive scalar, and therefore eddy diffusivity can only exceed molecular diffusivity (Biferale et al. , 1995). Thus the question, whether time-dependent flows can give rise to negative magnetic eddy diffusivity, is not trivial.

However, dynamo simulations for time-dependent turbulent flows are numerically demanding. For this reason we focus our attention here at an "intermediate" class of flows - those periodic in time, and employ the simplest of them:

$$
\mathbf{v}(\mathbf{x}, t)=\mathbf{U}(\mathbf{x})+\sqrt{\omega}\left(\mathbf{V}_{c}(\mathbf{x}) \cos \omega t+\mathbf{V}_{s}(\mathbf{x}) \sin \omega t\right) .
$$

Like in the cited papers, a flow is supposed to be $2 \pi$-periodic in spatial variables $\mathbf{x}$ and parity-invariant, i.e.

$$
\mathbf{v}(\mathbf{x}, t)=-\mathbf{v}(-\mathbf{x}, t)
$$

(Consequently, its space-averaged helicity is zero, and no $\alpha$-effect is present.)

Two families of space- and time-periodic flows were closely examined in the context of fast kinematic magnetic dynamo theory. Both are generalisations of $\mathrm{ABC}$ flows, and flows from both families are constructed of a small number of spatial Fourier harmonics. A flow of the kind of "modulated waves" was considered by Otani (1993) and Childress \& Gilbert (1995); it belongs to the class (1). "Circularly polarised" flows were employed in simulations of Galloway \& Proctor (1992) and Galloway \& O'Brian (1993); nearintegrable flows of this kind were studied analytically by Ponty et al. (1993, 1995) and Childress \& Gilbert (1995). The assumed time dependence was responsible for chaotic behaviour of the flow trajectories (this is necessary for a dynamo to be fast), though the flows depended only on two spatial variables (for steady flows this rules out chaos). This gave an opportunity to separate out dependence on the third spatial coordinate and to make convincing computations for high magnetic Reynolds numbers $R_{m} \sim 10^{4}$ (Galloway \& Proctor, 1992; Galloway \& O'Brian, 1993). Circularly polarised non-integrable ABC flows depending on three spatial variables were considered by Brummell et al. $(1999,2001)$.

We consider here a class of flows which seem to be more physically realistic: like Zheligovsky et al. (2001), we employ flows with a random spatial harmonic composition and an exponentially decaying energy spectrum. (Random-harmonic flows with a slow - hyperbolic - energy spectrum decay were also explored ibid., but they were found to be less efficient generators of large-scale magnetic field; consequently, such flows are not investigated here.) They can be regarded as a "poor man's model" of turbulent flows. 
Ideally we would like to examine dynamo properties of a large number of sample flows from the class under consideration, so that to collect statistically sound information. Unfortunately, computations even in the case of the simple time dependence (1) are numerically expensive: evaluation of one instance of magnetic eddy diffusivity requires more than a day of a CPU of a Dec Alpha processor. Hence it appears possible to evaluate only a limited number of such instances. However, since spatial Fourier components of the flows employed in computations are chosen at random, one can hope that results of computations presented here are typical and attributable to a large variety of flows. (Note, that flows from the selected class seize to be typical if $\mathbf{V}_{c}=0$ or $\mathbf{V}_{s}=0$ : it is shown in Section 3 that a non-zero contribution from the time-periodic part of (1) in the limit of high frequencies requires linear independence of the vector fields $\mathbf{V}_{c}$ and $\mathbf{V}_{s}$. Thus (1) represents the simplest class of time-periodic flows with hopefully a typical behaviour.)

Also due to numerical complexity of the problem, we have performed computations for just one value of molecular magnetic diffusivity, $\eta=0.1$. The following constraints restrict the choice of $\eta$ : On the one hand, if it is too large, diffusion dominates, inhibiting generation of magnetic field. On the other, if molecular diffusion is smaller than the threshold for the onset of generation of a small-scale magnetic field (i.e., magnetic field which has the same spatial periodicity, as the flow v), the short-scale instability dominates: the large-scale instability becomes "negligible", since growth rates of largescale magnetic modes are infinitesimally small (large-scale magnetic modes being perturbations of small-scale modes with non-vanishing spatial means, associated with a zero growth rate). Zheligovsky et al. (2001) explored largescale kinematic dynamos for three values of molecular magnetic diffusivity, $\eta=0.1,0.2$ and 0.3 (the flow was normalised so that its r.m.s. was 1 ). For all the three values no generation of small-scale magnetic field occurred, and for $\eta=0.1$ more flows sustained negative magnetic eddy diffusivity, than for $\eta=0.2$ and 0.3 . This has suggested to choose the value $\eta=0.1$ in our simulations; like ibid., we have checked that $\eta=0.1$ is above the magnetic diffusivity threshold for the onset of generation of a small-scale zero-mean magnetic field for every flow, for which magnetic eddy diffusivity has been computed.

In Section 1 we present a mathematical statement of the problem and state results of derivation of the magnetic eddy diffusivity tensor (details can be found in Appendix). Dependencies of the minimal magnetic diffusivity on the ratio of energies of the steady and time-dependent parts of the flow and on temporal frequency $\omega$ are studied numerically. Results of simulations are discussed in Section 2. Steady flows are found to be more capable of magnetic field generation, than time-periodic ones. Owing to the factor $\sqrt{\omega}$ in (1), in the limit of high temporal frequencies the time-dependent part of the flow provides a finite contribution to the magnetic eddy diffusivity tensor. In Section 3 the limit magnetic eddy diffusivity tensor is formally derived for $\omega \rightarrow \infty$, and it is shown numerically that flows can have negative eddy diffusivity for high frequencies. Our results are briefly summarized in the Conclusion. 


\section{Magnetic eddy diffusivity tensor for time-periodic flows}

In this Section we consider a kinematic dynamo problem for a flow $\mathbf{v}(\mathbf{x}, t)$ of time period $T$, which is $2 \pi$-periodic in each Cartesian variable in space, solenoidal $(\nabla \cdot \mathbf{v}=0)$ and parity-invariant $(2)$.

Temporal evolution of a magnetic field $\mathbf{h}$ is described by the magnetic induction equation

$$
\frac{\partial \mathbf{h}}{\partial t}=\eta \nabla^{2} \mathbf{h}+\nabla \times(\mathbf{v} \times \mathbf{h})
$$

Substituting $\mathbf{h}=\mathbf{H}(\mathbf{x}, t) e^{\lambda t}$ into (3) one finds that a magnetic mode $\mathbf{H}(\mathbf{x}, t)$ satisfies

$$
\lambda \mathbf{H}=-\frac{\partial \mathbf{H}}{\partial t}+\eta \nabla^{2} \mathbf{H}+\nabla \times(\mathbf{v} \times \mathbf{H})
$$

i.e. the kinematic dynamo problem reduces to a Floquet problem for the magnetic induction operator. The mode $\mathbf{H}(\mathbf{x}, t)$ is solenoidal,

$$
\nabla \cdot \mathbf{H}=0
$$

and it is assumed to have the same time period $T$, as the flow. $\operatorname{Re} \lambda$ is then the average rate of growth (or decay) of the mode in time.

We consider magnetic modes, involving large spatial scales. The modes are supposed to depend on the fast spatial variable $\mathbf{x}$ and on the slow variable $\mathbf{y}=\epsilon \mathbf{x}$. By the chain rule, spatial derivatives in the eigenmode equation (4) and the solenoidality condition (5) must be modified:

$$
\nabla \rightarrow \nabla_{\mathbf{x}}+\epsilon \nabla_{\mathbf{y}}
$$

(the subscripts $\mathbf{x}$ and $\mathbf{y}$ refer to differentiation in fast and slow variables, respectively). The ratio of the two scales, $\epsilon>0$, is a small parameter of the problem.

Solution to the Floquet problem (4) is sought in the form of power series

$$
\begin{gathered}
\lambda=\sum_{n=0}^{\infty} \lambda_{n} \epsilon^{n}, \\
\mathbf{H}=\sum_{n=0}^{\infty} \tilde{\mathbf{H}}_{n}(\mathbf{x}, \mathbf{y}, t) \epsilon^{n} .
\end{gathered}
$$

Substituting (6) and the series (7) and (8) into (4), expanding and equating coefficients for each power of $\epsilon$, one obtains a hierarchy of equations. It is discussed in Appendix, how all terms of (7) and (8) can be determined by a systematic procedure. In particular, it is shown that:

- The leading term in (7) is $\lambda_{2}$ and the expansion involves terms with even indices only $\left(\lambda_{0}=\lambda_{2 n+1}=0\right.$ for any integer $\left.n \geq 0\right)$.

- All terms of (8) with even indices are parity anti-invariant in fast variables $\left(\tilde{\mathbf{H}}_{2 n}(-\mathbf{x}, \mathbf{y}, t)=\tilde{\mathbf{H}}_{2 n}(\mathbf{x}, \mathbf{y}, t)\right.$ for any integer $\left.n \geq 0\right)$, and all terms with odd indices are parity-invariant in fast variables $\left(\tilde{\mathbf{H}}_{2 n+1}(-\mathbf{x}, \mathbf{y}, t)=-\tilde{\mathbf{H}}_{2 n+1}(\mathbf{x}, \mathbf{y}, t)\right.$ for any integer $n \geq 0$ ). 
- The leading term in the decomposition of an eigenmode is independent of time: $\tilde{\mathbf{H}}_{0}=\tilde{\mathbf{H}}_{0}(\mathbf{x}, \mathbf{y})$. Its spatial average satisfies the eigenvalue equation for the anisotropic magnetic eddy diffusivity operator,

$$
\eta \nabla_{\mathbf{y}}^{2}\left\langle\tilde{\mathbf{H}}_{0}\right\rangle+\nabla_{\mathbf{y}} \times \sum_{k=1}^{3} \sum_{m=1}^{3} \mathbf{D}_{m, k} \frac{\partial\left\langle\tilde{\mathbf{H}}_{0}^{k}\right\rangle}{\partial y_{m}}=\lambda_{2}\left\langle\tilde{\mathbf{H}}_{0}\right\rangle,
$$

and the solenoidality condition

$$
\nabla_{\mathbf{y}} \cdot\left\langle\tilde{\mathbf{H}}_{0}\right\rangle=0
$$

Here $\langle\cdot\rangle$ denotes the mean of a vector field over the cube of periodicity:

$$
\langle\mathbf{f}\rangle \equiv(2 \pi)^{-3} \int_{[0,2 \pi]^{3}} \mathbf{f}(\mathbf{x}, \mathbf{y}) d \mathbf{x}
$$

and superscripts enumerate Cartesian components of a vector field.

Coefficients of the eddy diffusivity tensor can be evaluated using solutions of two auxiliary problems:

$$
-\frac{\partial \mathbf{S}_{k}}{\partial t}+\eta \nabla^{2} \mathbf{S}_{k}+\nabla \times\left(\mathbf{v} \times \mathbf{S}_{k}\right)+\frac{\partial \mathbf{v}}{\partial x_{k}}=\mathbf{0}
$$

(the first auxiliary problem), and

$$
-\frac{\partial \boldsymbol{\Gamma}_{m, k}}{\partial t}+\eta \nabla^{2} \boldsymbol{\Gamma}_{m, k}+\nabla \times\left(\mathbf{v} \times \boldsymbol{\Gamma}_{m, k}\right)+2 \eta \frac{\partial \mathbf{S}_{k}}{\partial x_{m}}+\mathbf{e}_{m} \times\left(\mathbf{v} \times\left(\mathbf{S}_{k}+\mathbf{e}_{k}\right)\right)=\mathbf{0}
$$

(the second auxiliary problem); here $\left\{\mathbf{e}_{m}\right\}$ is the basis of Cartesian unit vectors. Then

$$
\mathbf{D}_{m, k}=\frac{1}{T} \int_{0}^{T}\left\langle\mathbf{v} \times \boldsymbol{\Gamma}_{m, k}\right\rangle d t
$$

It can be verified that $\mathbf{S}_{k}$ are parity anti-invariant $\left(\mathbf{S}_{k}(\mathbf{x}, t)=\mathbf{S}_{k}(-\mathbf{x}, t)\right)$ and solenoidal; $\boldsymbol{\Gamma}_{m, k}$ are parity-invariant and satisfy $\nabla_{\mathbf{x}} \cdot \boldsymbol{\Gamma}_{m, k}+\mathbf{S}_{k}^{m}=0$.

The partial differential operator in the left-hand side of (9) is comprised of second-order derivatives with constant coefficients. Consequently, its eigenvectors are Fourier harmonics: $\left\langle\tilde{\mathbf{H}}_{0}\right\rangle=\tilde{\mathbf{h}} e^{i \mathbf{q y}}$, where $\mathbf{q}$ is a (constant) wavevector, and $\tilde{\mathbf{h}}$ satisfies

$$
\begin{gathered}
\mathbf{q} \times \sum_{k=1}^{3} \sum_{m=1}^{3} \mathbf{D}_{m, k} \mathbf{q}_{m} \tilde{\mathbf{h}}_{k}+\eta|\mathbf{q}|^{2} \tilde{\mathbf{h}}=-\lambda_{2} \tilde{\mathbf{h}}, \\
\tilde{\mathbf{h}} \cdot \mathbf{q}=0 .
\end{gathered}
$$

The quantity $\eta_{\text {eddy }}=\min _{|\mathbf{q}|=1}\left(-\lambda_{2}\right)$ is regarded as the minimal magnetic eddy diffusivity. When it is negative, the associated magnetic mode grows in time. 


\section{Magnetic eddy diffusivity for flows (1): numerical results}

Numerical simulations have been carried out for flows (1), where $\mathbf{U}, \mathbf{V}_{c}$ and $\mathbf{V}_{s}$ are $2 \pi$-periodic parity-invariant solenoidal fields. The fields have been generated by the procedure which was applied by Zheligovsky et al. (2001): (i) a half of Fourier harmonics with random uniformly distributed components are generated (all the rest ones are obtained by complex conjugation, so that the vector field is real), (ii) the gradient part of the resultant field is projected out, and (iii) the harmonics are rescaled in each Fourier spherical shell to obtain the desirable energy spectrum. The spectrum of flows employed in our simulations exponentially decreases by 6 orders of magnitude, the Fourier series being truncated at wavenumber 10 . The vector fields are normalised so that

$$
E_{\text {total }}=1
$$

where

$$
E_{\text {total }} \equiv \frac{1}{T} \int_{0}^{T}(2 \pi)^{-3} \int_{[0,2 \pi]^{3}}|\mathbf{v}|^{2} d \mathbf{x} d t=\int_{[0,2 \pi]^{3}}\left(|\mathbf{U}|^{2}+\frac{1}{2}\left(\left|\mathbf{V}_{c}\right|^{2}+\left|\mathbf{V}_{s}\right|^{2}\right)\right) d \mathbf{x}
$$

is the average total energy of the flow for $\omega=1$ (hence the magnetic Reynolds number can be estimated as $R_{m}=\eta^{-1}$ ). Equipartition of the average energy of the two time-dependent terms in (1) is assumed:

$$
\int_{[0,2 \pi]^{3}}\left|\mathbf{V}_{c}\right|^{2} d \mathbf{x}=\int_{[0,2 \pi]^{3}}\left|\mathbf{V}_{s}\right|^{2} d \mathbf{x} .
$$

Solutions to the auxiliary problems (11) and (12) are sought in the form of Fourier series, with the $64^{3}$ Fourier harmonics resolution in space and 8 harmonics resolution in time. For this resolution the spatial energy spectra of the solutions decay by at least 10 orders of magnitude, and the temporal ones - by $4-5$ orders of magnitude.

All computations presented in this paper have been performed for molecular magnetic diffusivity $\eta=0.1$. It has been verified that for each sample flow (1), for which a magnetic eddy diffusivity value is reported here, the real part of the dominant eigenvalue of the magnetic induction operator acting in the space of $2 \pi$-periodic small-scale (i.e. independent of the slow variables) zero-mean magnetic fields is negative, i.e. $\eta=0.1$ is above the magnetic diffusivity threshold for the onset of generation of a small-scale magnetic field. Codes of Zheligovsky (1993a) have been applied for numerical treatment of the Floquet problems ${ }^{3}$ for small-scale magnetic modes.

The following questions have been addressed.

a) How does the minimal magnetic eddy diffusivity $\eta_{\text {eddy }}$ change when the flow (1) is close to a steady one? Magnetic eddy diffusivity has been evaluated for

\footnotetext{
${ }^{3}$ Note that in the Floquet problem (4) eigenmodes are defined up to a factor $e^{i J \omega t}$, where $J$ is an arbitrary integer, the associated eigenvalues differing by $i J \omega$. Hence in computations precautions must be taken to converge to the dominant eigenvalue, whose imaginary part does not exceed $\omega / 2$ in absolute value, i.e. whose imaginary part is minimal in absolute value in this set of equivalent eigenmodes.
} 
temporal frequency $\omega=1$ and molecular viscosity $\eta=0.1$ for 30 independent samples of (1) satisfying (15) and (16), such that the ratio of the average energy of the time-dependent part of (1),

$$
E_{\mathrm{osc}}=\frac{1}{2} \int_{[0,2 \pi]^{3}}\left(\left|\mathbf{V}_{c}\right|^{2}+\left|\mathbf{V}_{s}\right|^{2}\right) d \mathbf{x}
$$

to the energy of the steady profile is small:

$$
E_{\text {osc }} / \int_{[0,2 \pi]^{3}}|\mathbf{U}|^{2} d \mathbf{x}=1 / 400 .
$$

A histogram of the values of $\delta \eta_{\text {eddy }}$, the amount of change in magnetic eddy diffusivity due to introduction of this weak time periodicity into the flow, is shown on Fig. 1. Only in 2 cases out of 30 the moderate time dependence makes $\eta_{\text {eddy }}$ to decrease.

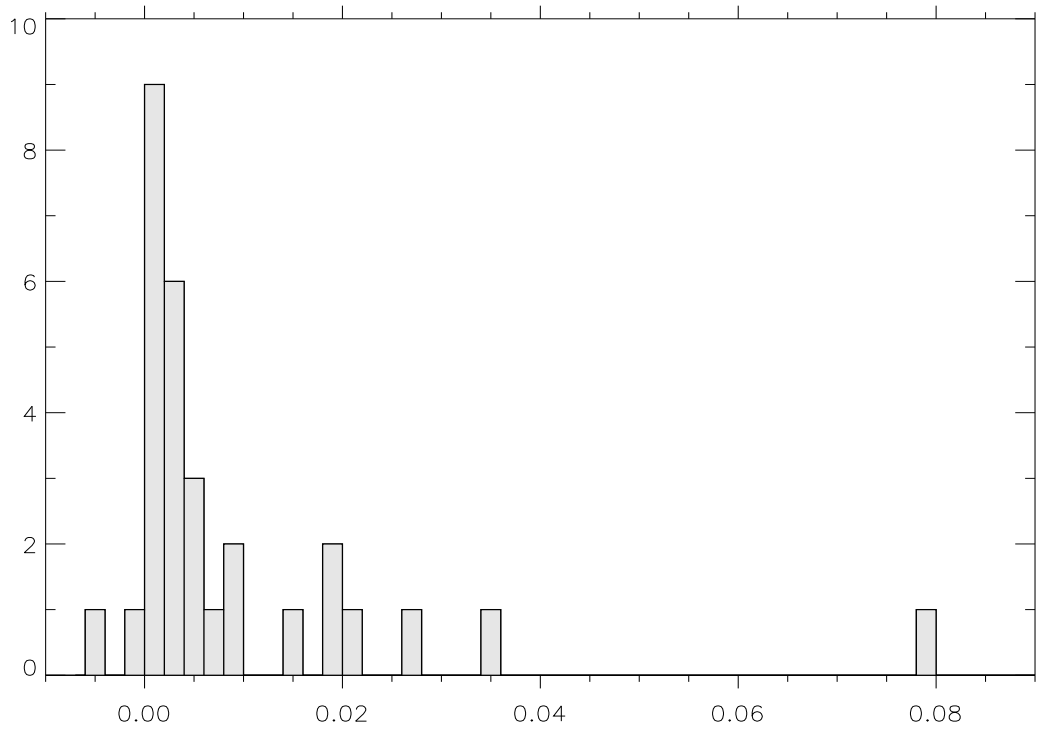

Figure 1. Histogram of values of $\delta \eta_{\text {eddy }}$, amounts of change in magnetic eddy diffusivity due to introduction of weak time periodicity into the flow.

b) How does minimal magnetic eddy diffusivity depend on how kinetic energy is split between the steady and time-dependent parts of the flow? Magnetic eddy diffusivity has been evaluated for three sets of sample flows (1) for $\omega=1$. Profiles of the constituent fields $\mathbf{U}(\mathbf{x}), \mathbf{V}_{c}(\mathbf{x})$ and $\mathbf{V}_{s}(\mathbf{x})$ are the same in each set, and only amplitudes of these fields are varied in such a way that (15) and (16) is satisfied. (In fact, in order to compute any of the three curves, a sample flow has been chosen out of those used to construct Fig. 1, and profiles of its constituent fields have been employed.)

Graphs of $\eta_{\text {eddy }}$ versus the ratio $E_{\text {osc }} / E_{\text {total }}$ are plotted on Fig. 2. Though graphs representing different flows are quite different in details, Fig. 2 reveals a common tendency: a relative increase of energy contained in the timedependent part of the flow is in general accompanied by an overall (although not necessarily monotonous) increase of magnetic eddy diffusivity. 


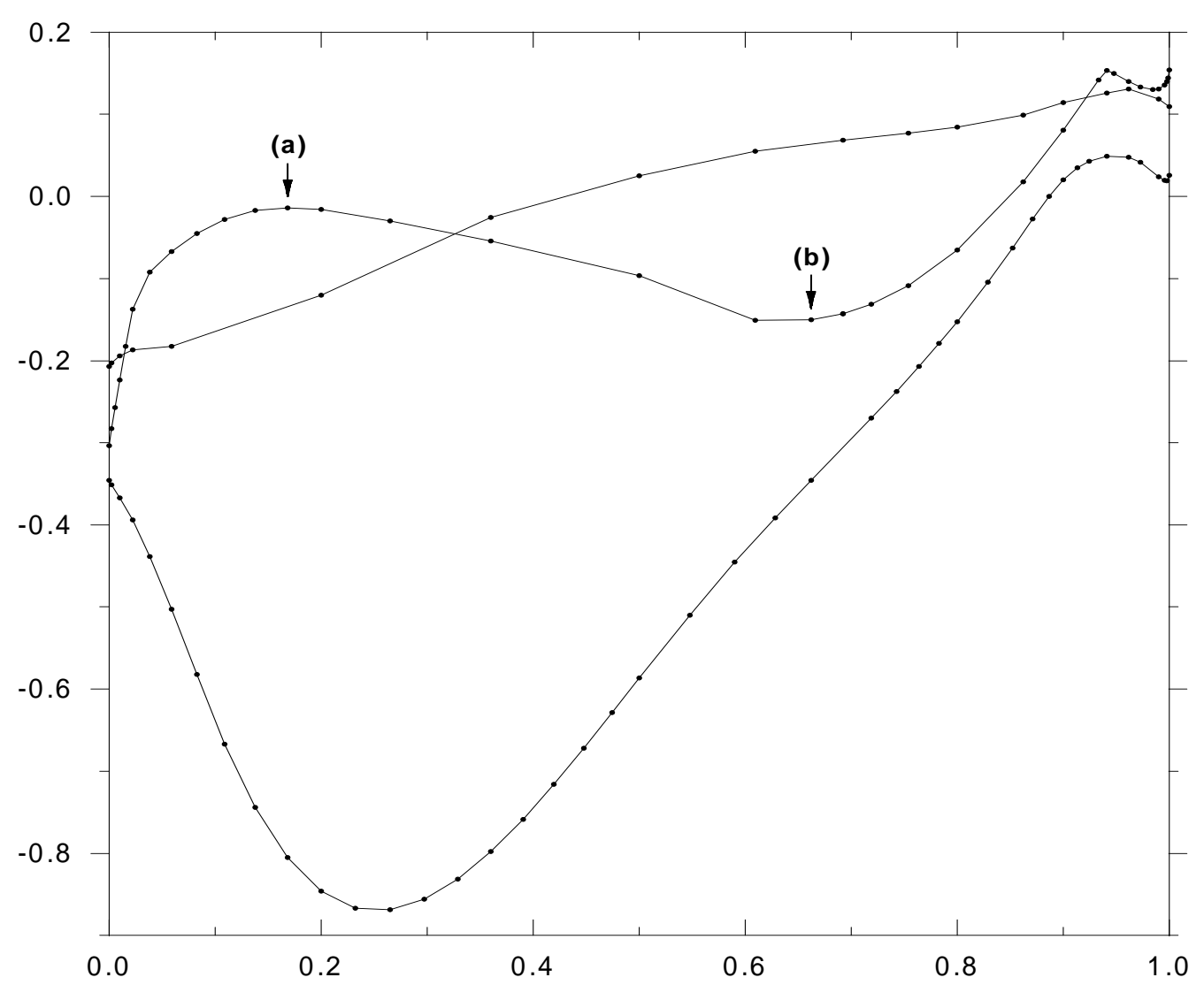

Figure 2. Minimal magnetic eddy diffusivity $\eta_{\text {eddy }}$ (vertical axis) as a function of the ratio $E_{\text {osc }} / E_{\text {total }}$ (horizontal axis) for three sets (represented by three curves) of sample flows (1) for $\omega=1$. Dots show computed values of magnetic eddy diffusivity. Two dots marked by arrows have been obtained for sample flows, for which results of computations are shown on Fig. 3.

Though time periodicity may be expected to enhance chaotic properties of flows (which are necessary for fast dynamo action), our results suggest that time dependence such as (1) is not favourable for generation of magnetic field. However, dynamos considered here are slow, and therefore this does not represent a formal contradiction.

c) How does magnetic eddy diffusivity depend on temporal frequency of the flow? Computations have been carried out for two flows (Fig. 3), which have the same profiles of the constituent fields $\mathbf{U}(\mathbf{x}), \mathbf{V}_{c}(\mathbf{x})$ and $\mathbf{V}_{s}(\mathbf{x})$. Two curves on Fig. 3, computed for the two flows, are labelled (a) and (b) in agreement with labelling of the two respective points on Fig. 2 (indicated on Fig. 2 by arrows). Different patterns of behaviour of magnetic eddy diffusivity are observed, though the two flows differ only in amplitudes of their steady and time-dependent parts. When $\omega \rightarrow \infty$, magnetic eddy diffusivity approaches finite limit values which can be significantly higher than $\min _{\omega} \eta_{\text {eddy }}(\omega)$, as it happens in the case (b) (in this case the sample flow has a relatively larger time-dependent part of the flow). The plots show that the minimal value of $\eta_{\text {eddy }}$ is achieved at moderate values of temporal frequency, $\omega=O(1)$. 


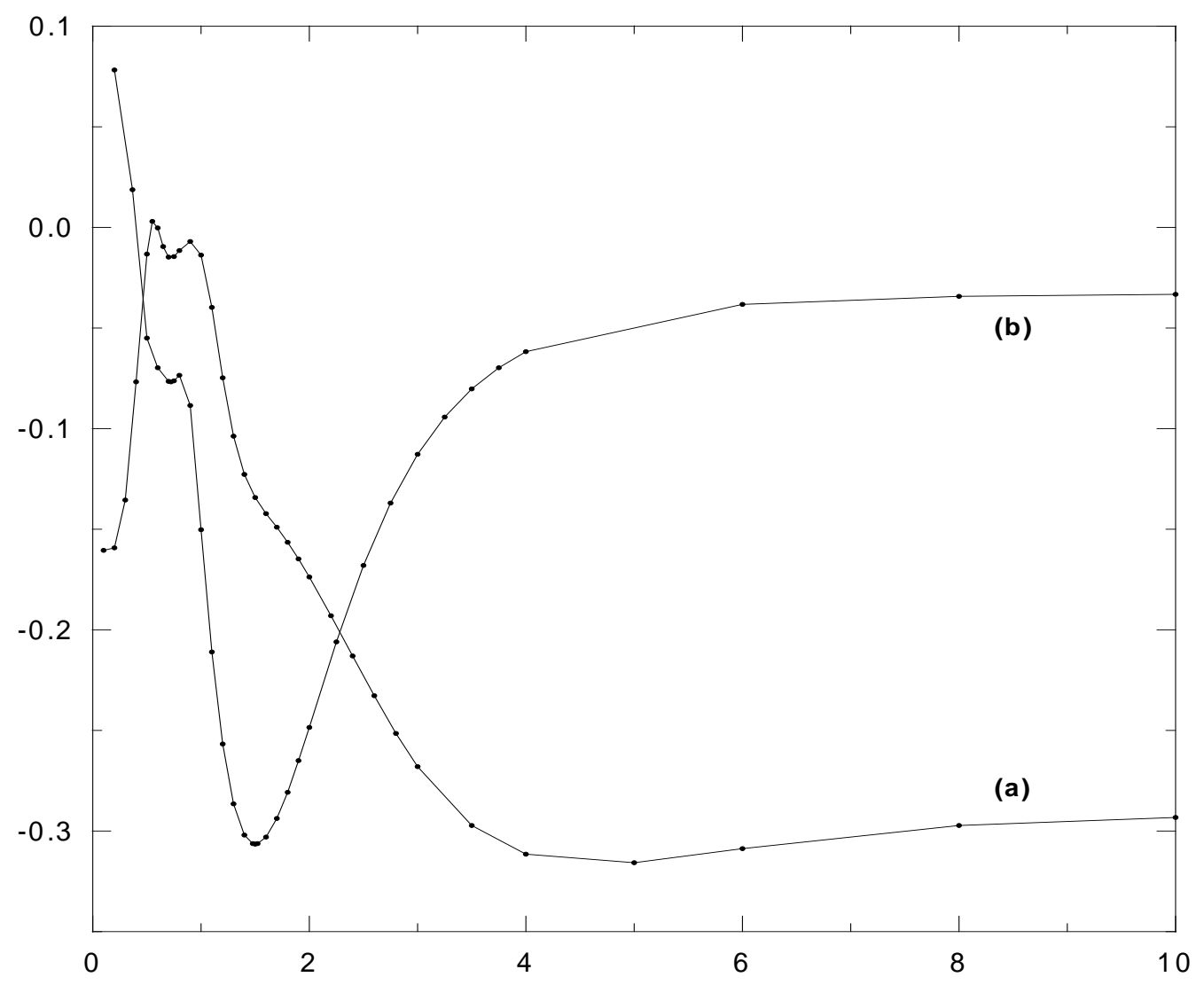

Figure 3. Minimal magnetic eddy diffusivity, $\eta_{\text {eddy }}$, (vertical axis) as a function of temporal frequency of the flow, $\omega$, (horizontal axis). Dots show computed values of magnetic eddy diffusivity. Curves are labelled (a) and (b), referring to the labels of the respective points for $\omega=1$ on Fig. 2 .

An example of a magnetic mode structure is shown on Fig. 4 and 5. The leading term in the expansion of a magnetic mode in the ratio of spatial scales, $\epsilon$, is

$$
\tilde{\mathbf{H}}_{0}=e^{i \epsilon \mathbf{q} \cdot \mathbf{x}}\left(\tilde{\mathbf{h}}+\sum_{k=1}^{3} \tilde{\mathbf{h}}^{k} \mathbf{S}_{k}(\mathbf{x}, t)\right)
$$

(see (A6), (A12) and (A22) in the Appendix), where $\mathbf{S}_{k}(\mathbf{x}, t)$ is the solution to the first auxiliary problem (11), and $\tilde{\mathbf{h}}$ is an eigenvector of the problem (13)-(14). The fluctuating part of the magnetic mode,

$$
\mathbf{G}=\sum_{k=1}^{3} \tilde{\mathbf{h}}^{k} \mathbf{S}_{k}(\mathbf{x}, t),
$$

is shown on Figs. 4 and 5 as surfaces of constant magnetic energy $|\mathbf{G}|^{2}$. The Figures show the magnetic mode generated by that flow, for which the curve (b) is shown on Fig. 3, for $\omega=1.5$, where the minimum of magnetic eddy diffusivity is located on this curve. Cigar-like magnetic structures, seen on the Figures, can be associated with stagnation points of the flow (1), though no flux-rope solutions similar to those of Zheligovsky (1993b) and Galloway and Zheligovsky (1994) are available for time-dependent flows. 

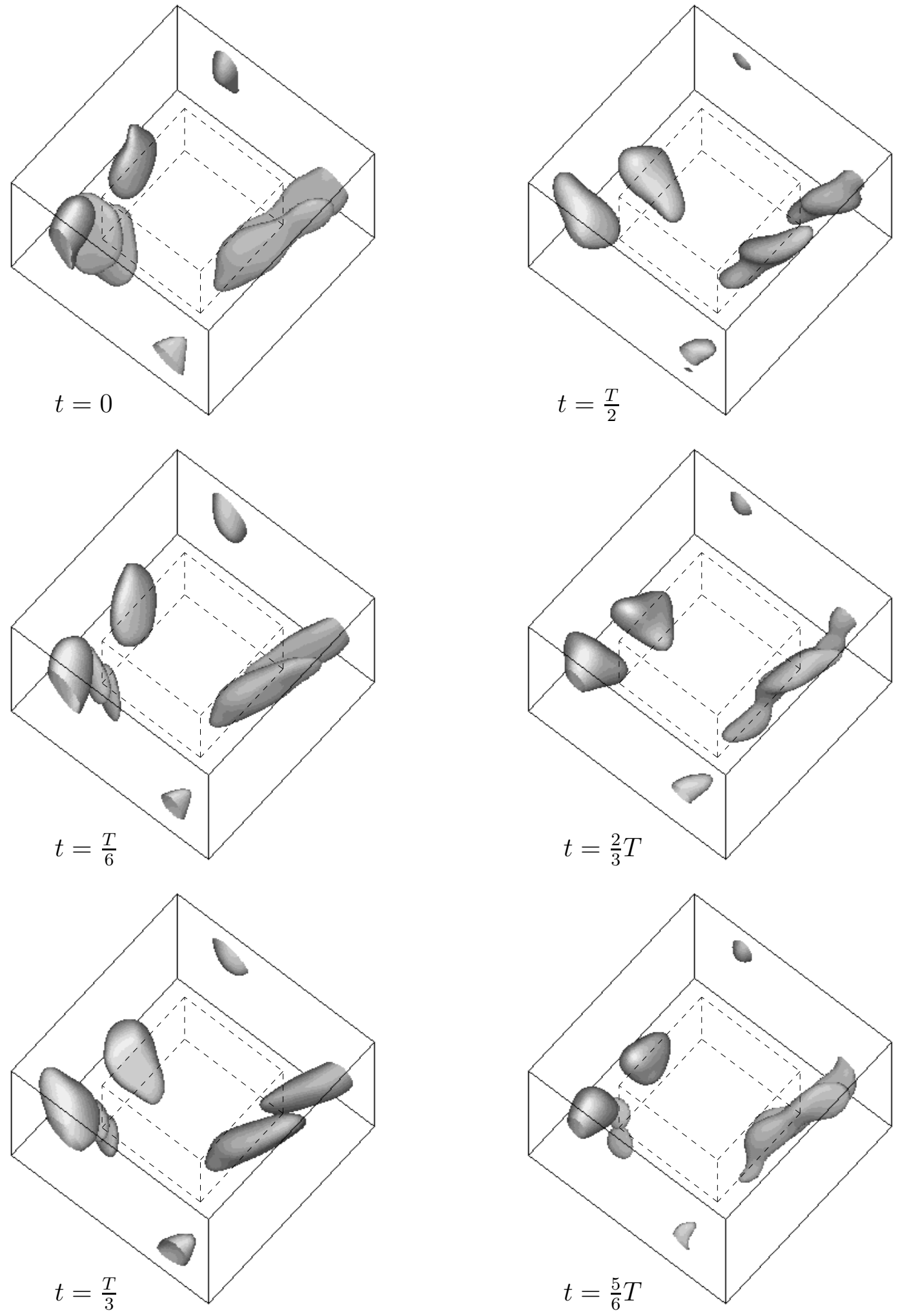

Figure 4. Isosurfaces of magnetic energy $|\mathbf{G}|^{2}$ of the fluctuating part of a magnetic mode at the level of $40 \%$ of the maximal energy. Snapshots of a flow periodicity cube (drawn in solid lines) step $T / 6$ are presented. The lower vertex is at the point $(-\pi / 2,-\pi / 2,-\pi / 2)$. Dashed lines show the elementary cube of the flow stagnation points mesh $\left(m_{1} \pi, m_{2} \pi, m_{3} \pi\right)$. 

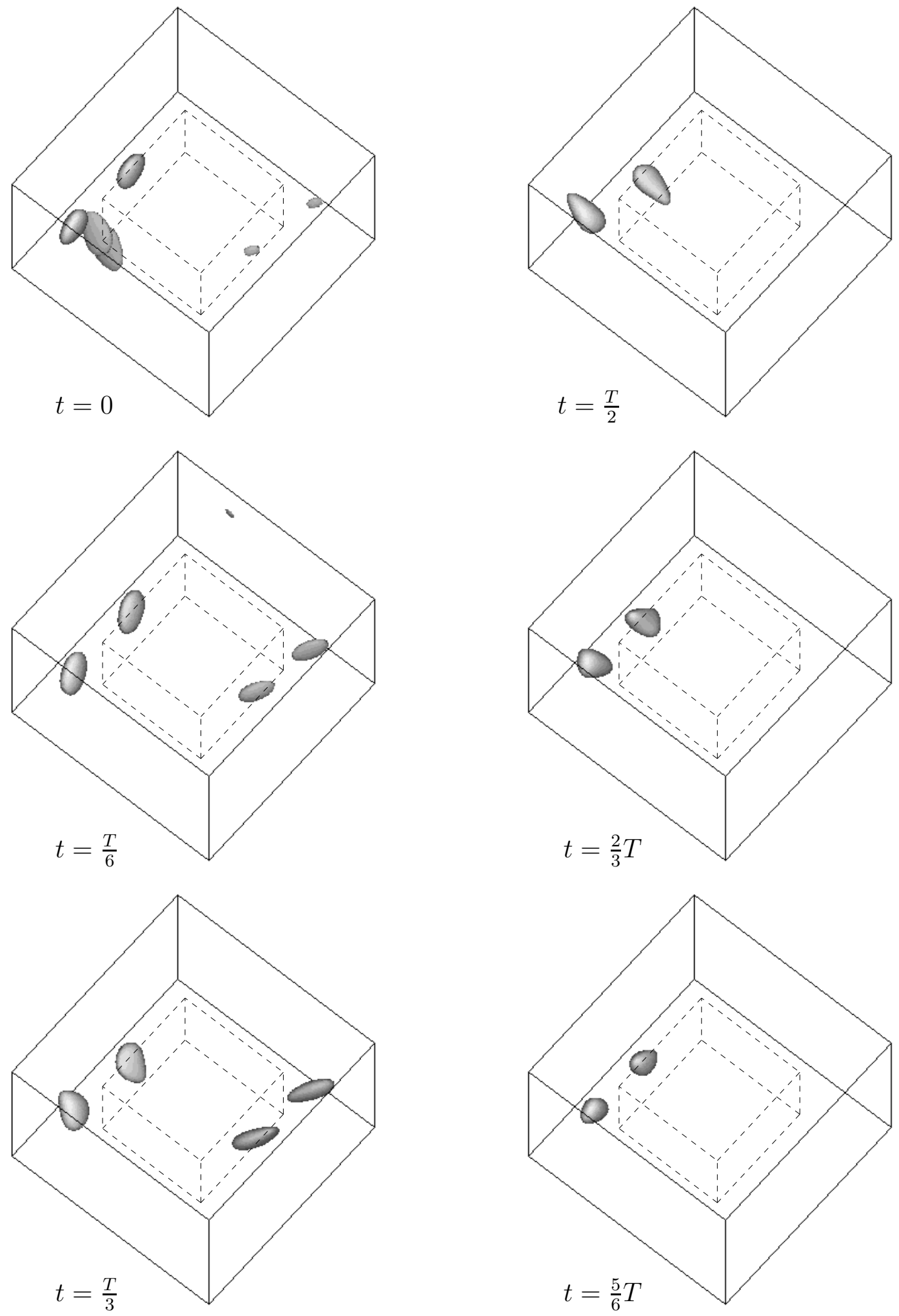

Figure 5. Same as Fig. 4, but isosurfaces of magnetic energy of the fluctuating part of the magnetic mode at the level of $75 \%$ of the maximal energy are shown. 
(Note, that due to parity invariance (2) and space periodicity of the flow with the period $2 \pi$ along each Cartesian axis, the flow velocity vanishes at the points $\left(m_{1} \pi, m_{2} \pi, m_{3} \pi\right)$, where $m_{1}, m_{2}, m_{3}$ are arbitrary integers.) The sharpness and the shape of the cigar structures vary considerably within the time period.

\section{Magnetic eddy diffusivity of flows (1) in the high frequency limit}

Figure 3 suggests, that in the limit $\omega \rightarrow \infty$ a contribution to magnetic eddy diffusivity from the small-scale dynamics of a flow (1) is finite. The limit of high frequencies is studied in this Section.

It is convenient to express the flow (1) in the form

$$
\mathbf{v}(\mathbf{x}, t)=\mathbf{U}(\mathbf{x})+\sqrt{\omega}\left(\mathbf{V}(\mathbf{x}) e^{i \omega t}+\overline{\mathbf{V}}(\mathbf{x}) e^{-i \omega t}\right),
$$

where $\mathbf{V}=\left(\mathbf{V}_{c}+i \mathbf{V}_{s}\right) / 2$. Denote by $\mathcal{L}$ the parabolic magnetic induction operator, acting in the space of $2 \pi / \omega$-periodic small-scale fields $\mathbf{F}(\mathbf{x}, t)$ with a zero mean $(\langle\mathbf{F}\rangle=0)$ :

$$
\mathcal{L} \mathbf{F} \equiv-\frac{\partial \mathbf{F}}{\partial t}+\eta \nabla^{2} \mathbf{F}+\nabla \times(\mathbf{v} \times \mathbf{F})
$$

Consider Fourier series of $\mathcal{L} \mathbf{F}$ :

$$
\mathcal{L} \mathbf{F}=\sum_{j=-\infty}^{\infty} \mathcal{L}_{j} \mathbf{F} e^{i j \omega t} .
$$

For the flow (17)

$$
\mathcal{L}_{j} \mathbf{F}=-i j \omega \mathbf{F}_{j}+\eta \nabla^{2} \mathbf{F}_{j}+\nabla \times\left(\mathbf{U} \times \mathbf{F}_{j}\right)+\sqrt{\omega}\left(\mathbf{V} \times \mathbf{F}_{j-1}+\overline{\mathbf{V}} \times \mathbf{F}_{j+1}\right),
$$

where $\mathbf{F}_{j}$ are temporal Fourier components of $\mathbf{F}$ :

$$
\mathbf{F}(\mathbf{x}, t)=\sum_{j=-\infty}^{\infty} \mathbf{F}_{j}(\mathbf{x}) e^{i j \omega t}
$$

In terms of the Fourier components, the first auxiliary problem takes the form

$$
\mathcal{L}_{j} \mathbf{S}_{k}=-\frac{\partial}{\partial x_{k}}\left(\delta_{0}^{j} \mathbf{U}+\sqrt{\omega}\left(\delta_{1}^{j} \mathbf{V}+\delta_{-1}^{j} \overline{\mathbf{V}}\right)\right)
$$

where $\delta$ is the Kronecker symbol, and the second one -

$$
\begin{gathered}
\mathcal{L}_{j} \boldsymbol{\Gamma}_{m, k}=-2 \eta \frac{\partial \mathbf{S}_{j, k}}{\partial x_{m}}+\mathbf{U}^{m}\left(\mathbf{S}_{j, k}+\delta_{0}^{j} \mathbf{e}_{k}\right)-\mathbf{U S}_{j, k}^{m} \\
+\sqrt{\omega}\left(\mathbf{V}^{m}\left(\mathbf{S}_{j-1, k}+\delta_{1}^{j} \mathbf{e}_{k}\right)+\overline{\mathbf{V}}^{m}\left(\mathbf{S}_{j+1, k}+\delta_{-1}^{j} \mathbf{e}_{k}\right)-\mathbf{V} \mathbf{S}_{j-1, k}^{m}-\overline{\mathbf{V}} \mathbf{S}_{j+1, k}^{m}\right),
\end{gathered}
$$

where $\mathbf{S}_{j, k}$ are Fourier components of $\mathbf{S}_{k}$ :

$$
\mathbf{S}_{k}(\mathbf{x}, t)=\sum_{j=-\infty}^{\infty} \mathbf{S}_{j, k}(\mathbf{x}) e^{i j \omega t}
$$


Assuming a power-law asymptotic behaviour of the Fourier components of $\mathbf{S}_{k}$ and $\boldsymbol{\Gamma}_{m, k}$, one finds

$$
\mathbf{S}_{j, k}=\omega^{-|j| / 2} \mathbf{s}_{j, k}+O\left(\omega^{-(|j|+1) / 2}\right)
$$

to be consistent with (18) and (19), and

$$
\boldsymbol{\Gamma}_{j, m, k}=\omega^{-|j| / 2} \gamma_{j, m, k}+O\left(\omega^{-(|j|+1) / 2}\right)
$$

is consistent with (18) and (20). Here $\boldsymbol{\Gamma}_{j, m, k}$ are Fourier components of $\boldsymbol{\Gamma}_{m, k}$ :

$$
\boldsymbol{\Gamma}_{m, k}(\mathbf{x}, t)=\sum_{j=-\infty}^{\infty} \boldsymbol{\Gamma}_{j, m, k}(\mathbf{x}) e^{i j \omega t}
$$

Leading terms of the Fourier series of $\mathbf{S}_{k}$ and $\boldsymbol{\Gamma}_{m, k}$ satisfy

$$
\begin{gathered}
\eta \nabla^{2} \mathbf{s}_{0, k}+\nabla_{\mathbf{x}} \times\left(2 \operatorname{Re}\left(\overline{\mathbf{V}} \times \mathbf{s}_{1, k}\right)+\mathbf{U} \times \mathbf{s}_{0, k}\right)=-\frac{\partial \mathbf{U}}{\partial x_{k}}, \\
\mathbf{s}_{1, k}=-i\left(\frac{\partial \mathbf{V}}{\partial x_{k}}+\nabla_{\mathbf{x}} \times\left(\mathbf{V} \times \mathbf{s}_{0, k}\right)\right) \\
\eta \nabla^{2} \boldsymbol{\gamma}_{0, m, k}+\nabla_{\mathbf{x}} \times\left(2 \operatorname{Re}\left(\overline{\mathbf{V}} \times \boldsymbol{\gamma}_{1, m, k}\right)+\mathbf{U} \times \gamma_{0, m, k}\right) \\
=-2 \eta \frac{\partial \mathbf{s}_{0, k}}{\partial x_{m}}+2 \operatorname{Re}\left(\overline{\mathbf{V}}^{m} \mathbf{s}_{1, k}-\overline{\mathbf{V}} \mathbf{s}_{1, k}^{m}\right) \\
\gamma_{1, m, k}=-i\left(\mathbf{V}^{m}\left(\mathbf{s}_{0, k}+\mathbf{e}_{k}\right)-\mathbf{V} \mathbf{s}_{0, k}^{m}+\nabla_{\mathbf{x}} \times\left(\mathbf{V} \times \gamma_{0, m, k}\right)\right) .
\end{gathered}
$$

Hence, for $\omega \rightarrow \infty$ coefficients of the eddy diffusivity tensor are

$$
\mathbf{D}_{m, k}=2 \operatorname{Re}\left\langle\overline{\mathbf{V}} \times \gamma_{1, m, k}\right\rangle+\left\langle\mathbf{U} \times \gamma_{0, m, k}\right\rangle+O\left(\omega^{-1 / 2}\right) .
$$

The limit values of $\mathbf{D}_{m, k}$ are not affected by phase shifts: (21) and (22) imply that if $\mathbf{V}$ is modified to become $e^{i \alpha} \mathbf{V}$, where $\alpha$ is a real constant, then $\mathbf{s}_{ \pm 1, k}$ changes to $e^{ \pm i \alpha} \mathbf{s}_{ \pm 1, k}, \gamma_{ \pm 1, m, k}-$ to $e^{ \pm i \alpha} \gamma_{ \pm 1, m, k}$, and $\mathbf{s}_{0, k}, \gamma_{0, m, k}$ and $\mathbf{D}_{m, k}$ remain unaltered. $\mathbf{s}_{0, k}$ and $\gamma_{0, m, k}$ are real; if also $\mathbf{V}$ is real (i.e. if $\mathbf{V}_{s}=\mathbf{0}$ ), then $\mathbf{s}_{ \pm 1, k}$ and $\gamma_{ \pm 1, m, k}$ are imaginary, and hence

$$
\operatorname{Re}\left(\overline{\mathbf{V}} \times \mathbf{s}_{1, k}\right)=\operatorname{Re}\left(\overline{\mathbf{V}} \times \gamma_{1, m, k}\right)=0 .
$$

Therefore, if $\operatorname{Im} \mathbf{V}=\mathbf{0}$, then in the limit $\omega \rightarrow \infty$ a contribution from the time-periodic part of the flow (17) vanishes. Combined together, these two observations imply that a non-zero contribution from the time-periodic part of (17) requires linear independence of vector fields $\mathbf{V}_{c}$ and $\mathbf{V}_{s}$.

The same value of molecular viscosity, $\eta=0.1$, has been employed in simulations. Short-scale magnetic modes can be analyzed in the limit $\omega \rightarrow \infty$ along the same lines, as above: their temporal Fourier components exhibit the same power-law asymptotical behaviour, as solutions to the auxiliary problems. The limit small-scale magnetic modes turn out to be eigenfunctions of the linear operator defined by the left-hand sides of (21) and (22). It has been verified that for each sample flow (1), for which a limit magnetic 
eddy diffusivity value is reported here, the limit magnetic induction operator acting in the space of $2 \pi$-periodic small-scale zero-mean magnetic fields has no eigenvalues with a positive real part, i.e. for the employed flows $\eta=0.1$ is above the threshold for the onset of generation of small-scale magnetic fields in the limit of high temporal frequencies.

We carried out the following numerical experiments:

a) Limit values of magnetic eddy diffusivities have been computed using (21) and (22) for those three sets of sample flows (1) (see Fig. 6), for which plots of Fig. 2 have been constructed for $\omega=1$. The left-most points (for $E_{\text {osc }}=0$ ) of two respective curves on Figs. 2 and 6, obtained for the same set of sample flows, show the same value; this can be used to identify pairs of the respective curves. Like for finite frequency (see Section 2), a relative increase of energy contained in the time-dependent part of the flow is accompanied by an overall increase of magnetic eddy diffusivity, though the dependence is not necessarily monotonous. However, the influence of time-dependent parts of flows has now weakened.

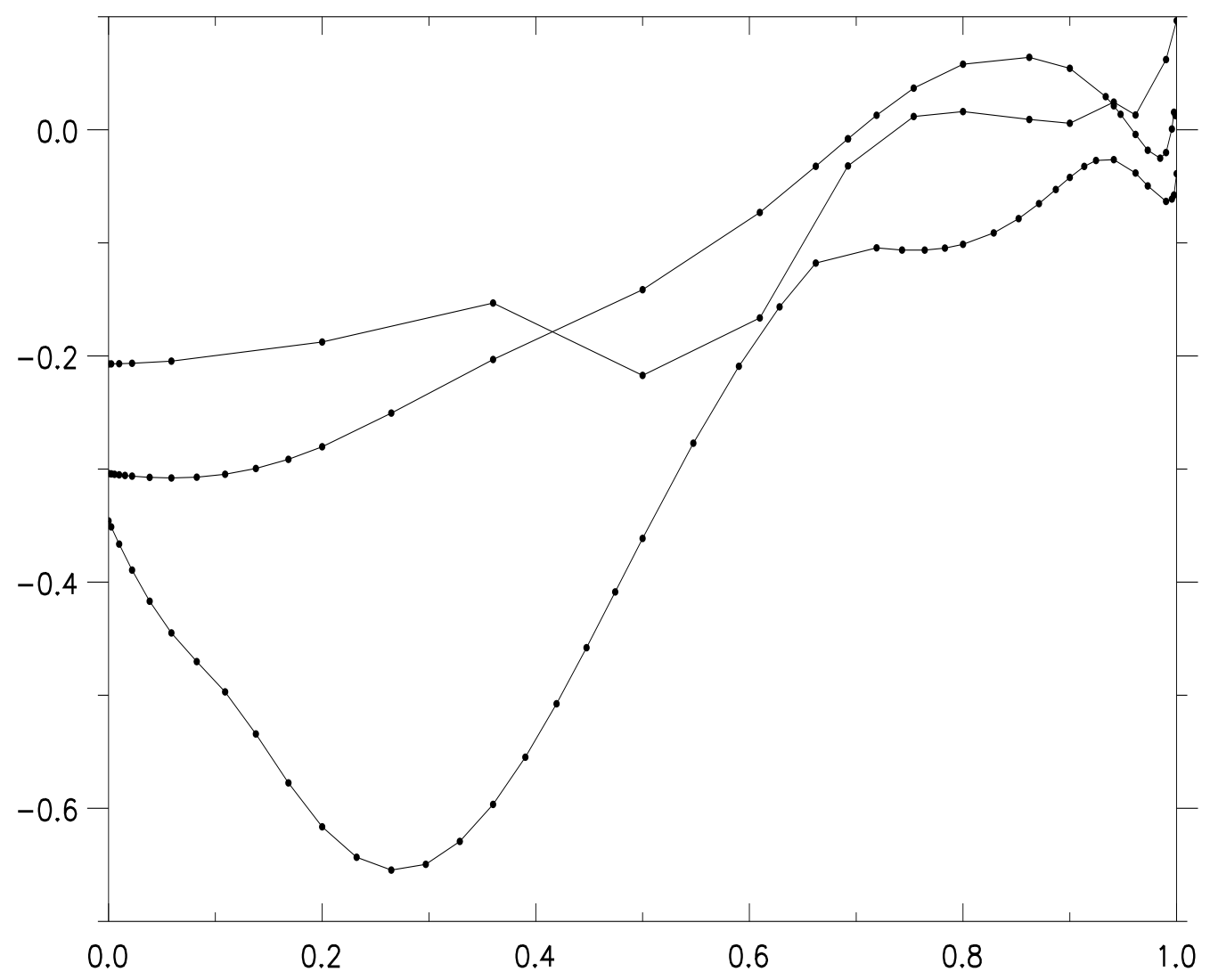

Figure 6. Minimal magnetic eddy diffusivity $\eta_{\text {eddy }}$ (vertical axis) in the limit $\omega \rightarrow \infty$ as a function of the ratio $E_{\text {osc }} / E_{\text {total }}$ (horizontal axis) for three sets (represented by three curves) of sample flows (1). Dots show computed values of magnetic eddy diffusivity. 
b) Distribution of minimal magnetic eddy diffusivity for (1) has been examined under the conditions, apparently least favourable for generation - in the absence of the steady component (i.e. for $\mathbf{U}=\mathbf{0}$ ) and in the limit $\omega \rightarrow \infty$. A histogram of limit values of $\eta_{\text {eddy }}$ computed for 45 sample flows, satisfying (15), (16) and $\mathbf{U}=\mathbf{0}$, is shown on Fig. 7. Only in 2 cases out of 45 the limit magnetic eddy diffusivity is negative.

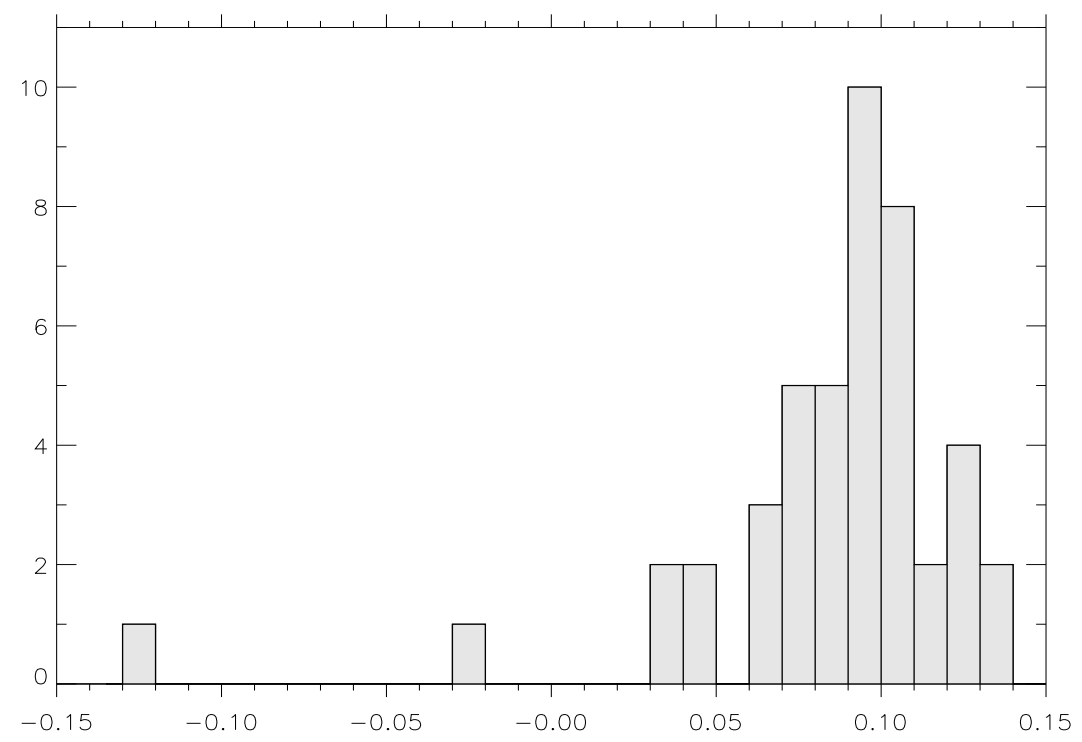

Figure 7. Histogram of minimal magnetic eddy diffusivity values in the limit $\omega \rightarrow \infty$ for 45 sample flows satisfying (15), (16) and $\mathbf{U}=\mathbf{0}$.

\section{Conclusion}

Generation of magnetic field involving large spatial scales by time- and space-periodic small-scale parity-invariant flows has been studied. The anisotropic magnetic eddy diffusivity tensor has been calculated. A complete expansion of magnetic modes and their growth rates in power series in the scale ratio has been constructed for flows (1). Simulations have been carried out for flows (1) with random harmonic composition and an exponentially decaying energy spectrum. Flows giving rise to negative magnetic eddy diffusivity have been found, for molecular diffusivity above the instability threshold for small-scale magnetic field generation. Numerical results suggest that generation of large-scale magnetic field by time-periodic flows is less efficient than generation by steady flows of the same energy. High temporal frequencies may also be unfavourable for generation. Nevertheless, we have found numerically instances of flows (1) with a vanishing steady component, for which magnetic eddy diffusivity is negative for $\omega \rightarrow \infty$. 


\section{Acknowledgments}

We are grateful to U. Frisch for extensive discussions. In the course of preparation of the final version of the paper we have benefited from the referees' remarks. A part of numerical results were obtained using computational facilities provided by the program "Simulations Interactives et Visualisation en Astronomie et Mécanique (SIVAM)" at Observatoire de la Côte d'Azur and by the Département Sciences Physiques pour l'Ingénieur of CNRS, France. Our research at Observatoire de la Côte d'Azur was supported by the French Ministry of Education. The investigation was concluded during our stay at the School of Mathematical Sciences, University of Exeter, UK, in May - July 2002. We are grateful to the Royal Society for their support of the visit.

\section{References}

Biferale L., Crisanti A., Vergassola M. and Vulpiani A. "Eddy diffusivity in scalar transport", Phys. Fluids 7, 2725-2734 (1995).

Brummell N.H., Cattaneo F. and Tobias S.M. "Nonlinear dynamo action in a time-dependent ABC flow", in Stellar dynamos: Nonlinearity and chaotic flows. Proc. of the International workshop held at Medina del Campo, Valladolid, Spain, 28-30 September, 1998 (Eds. M. Núñez and A. Ferriz-Mas). Astr. Soc. of the Pacific, Conf. series vol. 178, San Francisco, 23-34 (1999).

Brummell N.H., Cattaneo F. and Tobias S.M. "Linear and nonlinear dynamo properties of time-dependent ABC flows", Fluid Dynamics Res. 28, 237-265 (2001).

Childress S. and Gilbert A.D. Stretch, twist, fold: the fast dynamo, SpringerVerlag, Berlin (1995).

Galloway D.J. and O'Brian, N.R. "Numerical calculations of dynamos for ABC and related flows", in Solar and Planetary Dynamos (Eds. M.R.E. Proctor, P.C. Matthews, A.M. Rucklidge) Cambridge Univ. Press, 105-113 (1993).

Galloway D.J. and Proctor, M.R.E. "Numerical calculations of fast dynamos for smooth velocity fields with realistic diffusion", Nature 356, 691-693 (1992).

Galloway D.J. and Zheligovsky V.A. "On a class of non-axisymmetric flux rope solutions to the electromagnetic induction equation", Geophys. Astrophys. Fluid Dynamics, 76, 253-264 (1994).

Lanotte A., Noullez A., Vergassola M. and Wirth A. "Large-scale dynamo by negative magnetic eddy diffusivities", Geophys. Astrophys. Fluid Dynamics 91, 131-146 (1999). 
Otani N.F. "A fast kinematic dynamo in two-dimensional time-dependent flows", J. Fluid Mech. 253, 327-340 (1993).

Ponty Y., Pouquet A., Rom-Kedar A. and Sulem P.L. "Dynamo action in a nearly integrable chaotic flow", in Solar and Planetary Dynamos (Eds. M.R.E. Proctor, P.C. Matthews, A.M. Rucklidge) Cambridge Univ. Press, 241-248 (1993).

Ponty Y., Pouquet A. and Sulem P.L. "Dynamos in weakly chaotic twodimensional flows", Geophys. Astrophys. Fluid Dynamics 79, 239-257 (1995).

Zheligovsky V.A. "Numerical solution of the kinematic dynamo problem for Beltrami flows in a sphere", J. of Scientific Computing, 8, 41-68 (1993a).

Zheligovsky V.A. "A kinematic magnetic dynamo sustained by a Beltrami flow in a sphere", Geophys. Astrophys. Fluid Dynamics, 73, 217-254 (1993b).

Zheligovsky V.A., Podvigina O.M. and Frisch U. "Dynamo effect in parityinvariant flow with large and moderate separation of scales", Geophys. Astrophys. Fluid Dynamics 95, 227-268 [http://xxx.lanl.gov/abs/nlin.CD/0012005] (2001). 


\section{Appendix. Formal asymptotic decomposition of magnetic modes and of their growth rates for time-periodic flows (1)}

We derive complete asymptotic expansions of a magnetic mode and its growth rate for the flow (1), which it is convenient to express here as

$$
\mathbf{v}(\mathbf{x}, t)=\mathbf{U}(\mathbf{x})+\mathbf{W}(\mathbf{x}) e^{i \omega t}+\overline{\mathbf{W}}(\mathbf{x}) e^{-i \omega t},
$$

where the notation

$$
\mathbf{W}=\sqrt{\omega} \mathbf{V}
$$

is used. Our derivation follows that of Zheligovsky et al. (2001) for steady flows.

As in the main body of the paper, $\mathbf{x} \in R^{3}$ denotes the fast, and $\mathbf{y}=\epsilon \mathbf{x}$ - the slow spatial variable, $\langle\cdot\rangle$ and $\{\cdot\}$ denote the mean and the fluctuating part of a vector field, respectively:

$$
\langle\mathbf{f}(\mathbf{x}, \mathbf{y}, \mathbf{t})\rangle \equiv(2 \pi)^{-3} \int_{[0,2 \pi]^{3}} \mathbf{f}(\mathbf{x}, \mathbf{y}, t) d \mathbf{x}, \quad\{\mathbf{f}(\mathbf{x}, \mathbf{y}, t)\} \equiv \mathbf{f}-\langle\mathbf{f}\rangle .
$$

The following assumptions are made concerning the flow $\mathbf{v}$ : $\mathbf{U}$ and $\mathbf{W}$ are $2 \pi$-periodic in fast Cartesian variables, independent of time and of the slow variables, solenoidal:

$$
\nabla \cdot \mathbf{U}=\nabla \cdot \mathbf{W}=0
$$

and parity-invariant:

$$
\mathbf{U}(\mathbf{x})=-\mathbf{U}(-\mathbf{x}), \quad \mathbf{W}(\mathbf{x})=-\mathbf{W}(-\mathbf{x}) .
$$

A magnetic mode $\mathbf{H}(\mathbf{x}, \mathbf{y}, t)$ is a solenoidal (5) solution to the Floquet problem (4), which is $2 \pi$-periodic in each spatial variable and has the same temporal period $T=2 \pi / \omega$, as the flow. For the flow (A1), Fourier components of a magnetic mode

$$
\mathbf{H}(\mathbf{x}, \mathbf{y}, t)=\sum_{j=-\infty}^{\infty} \mathbf{H}_{j}(\mathbf{x}, \mathbf{y}) e^{i j \omega t}
$$

satisfy

$$
\lambda \mathbf{H}_{j}=-i j \omega \mathbf{H}_{j}+\eta \nabla^{2} \mathbf{H}_{j}+\nabla \times\left(\mathbf{W} \times \mathbf{H}_{j-1}+\mathbf{U} \times \mathbf{H}_{j}+\overline{\mathbf{W}} \times \mathbf{H}_{j+1}\right)
$$

for all $j$.

A solution to this system of equations is sought in the form of power series

$$
\mathbf{H}_{j}(\mathbf{x}, \mathbf{y})=\sum_{n=0}^{\infty}\left(\mathbf{H}_{j, n}(\mathbf{y})+\mathbf{G}_{j, n}(\mathbf{x}, \mathbf{y})\right) \epsilon^{n}
$$

and (7). In (A6) $\mathbf{H}_{j, n}$ and $\mathbf{G}_{j, n}$ are the mean and the fluctuating part of the respective term of the series: $\left\langle\mathbf{G}_{j, n}\right\rangle=0$. Obviously, any term $\tilde{\mathbf{H}}_{n}$ of the expansion (8) can be recovered from (A6):

$$
\tilde{\mathbf{H}}_{n}(\mathbf{x}, \mathbf{y}, t)=\sum_{j=-\infty}^{\infty}\left(\mathbf{H}_{j, n}(\mathbf{y})+\mathbf{G}_{j, n}(\mathbf{x}, \mathbf{y})\right) e^{i j \omega t}
$$


After modification of the spatial gradient (6), expansion and separation of mean and fluctuating parts of each Fourier harmonics, the solenoidality condition (5) reduces to

$$
\begin{gathered}
\nabla_{\mathbf{y}} \cdot \mathbf{H}_{j, n}=0 \\
\nabla_{\mathbf{x}} \cdot \mathbf{G}_{j, n}+\nabla_{\mathbf{y}} \cdot \mathbf{G}_{j, n-1}=0
\end{gathered}
$$

for all $j$ and $n \geq 0$, where it is understood $\mathbf{G}_{j, n} \equiv 0$ for $n<0$. Here and in what follows the subscripts $\mathbf{x}$ and $\mathbf{y}$ refer to differential operators in fast and slow variables, respectively.

Substitution of (A6), (7) and (6) into (A5) yields

$$
\begin{gathered}
\sum_{n=0}^{\infty}\left[-i j \omega \mathbf{H}_{j, n}+\mathcal{L}_{j} \mathbf{G}_{\cdot, n}+\eta\left(2\left(\nabla_{\mathbf{x}} \cdot \nabla_{\mathbf{y}}\right) \mathbf{G}_{j, n-1}+\nabla_{\mathbf{y}}^{2}\left(\mathbf{H}_{j, n-2}+\mathbf{G}_{j, n-2}\right)\right)\right. \\
\quad+\nabla_{\mathbf{x}} \times\left(\mathbf{W} \times \mathbf{H}_{j-1, n}+\mathbf{U} \times \mathbf{H}_{j, n}+\overline{\mathbf{W}} \times \mathbf{H}_{j+1, n}\right) \\
\quad+\nabla_{\mathbf{y}} \times\left(\mathbf{W} \times\left(\mathbf{H}_{j-1, n-1}+\mathbf{G}_{j-1, n-1}\right)+\mathbf{U} \times\left(\mathbf{H}_{j, n-1}+\mathbf{G}_{j, n-1}\right)\right. \\
\left.\left.+\overline{\mathbf{W}} \times\left(\mathbf{H}_{j+1, n-1}+\mathbf{G}_{j+1, n-1}\right)\right)-\sum_{m=0}^{n} \lambda_{n-m}\left(\mathbf{H}_{j, m}+\mathbf{G}_{j, m}\right)\right] \epsilon^{n}=0 .
\end{gathered}
$$

Here it is denoted

$$
\mathcal{L}_{j} \mathbf{F} \equiv \eta \nabla_{\mathbf{x}}^{2} \mathbf{F}_{j}+\nabla_{\mathbf{x}} \times\left(\mathbf{W} \times \mathbf{F}_{j-1}+\mathbf{U} \times \mathbf{F}_{j}+\overline{\mathbf{W}} \times \mathbf{F}_{j+1}\right)-i j \omega \mathbf{F}_{j},
$$

where $\mathbf{F}_{j}$ are Fourier components of

$$
\mathbf{F}(\mathbf{x}, \mathbf{y}, t)=\sum_{j=-\infty}^{\infty} \mathbf{F}_{j}(\mathbf{x}, \mathbf{y}) e^{i j \omega t}
$$

We make a final technical assumption that for any function $\mathbf{f}(\mathbf{x}, t)$, $T$-periodic in time and $2 \pi$-periodic in space, such that $\langle\mathbf{f}\rangle=0$ for all $t$, the problem $\mathcal{L} \mathbf{F}=\mathbf{f}$ has a unique small-scale solenoidal solution with a vanishing spatial mean, which has the same time and space periodicities, as the flow. (Equivalently, the magnetic induction operator $\mathcal{L}$ is assumed to have a trivial kernel.) Generically this condition holds.

We proceed by successively equating the mean and the fluctuating part of each term of the series (A8) to zero.

$i$. The leading $(n=0)$ term of (A8) takes the form

$$
\begin{gathered}
\mathcal{L}_{j} \mathbf{G}_{\cdot, 0}+\left(\mathbf{H}_{j-1,0} \cdot \nabla_{\mathbf{x}}\right) \mathbf{W}+\left(\mathbf{H}_{j, 0} \cdot \nabla_{\mathbf{x}}\right) \mathbf{U}+\left(\mathbf{H}_{j+1,0} \cdot \nabla_{\mathbf{x}}\right) \overline{\mathbf{W}} \\
=\lambda_{0}\left(\mathbf{H}_{j, 0}+\mathbf{G}_{j, 0}\right)+i j \omega \mathbf{H}_{j, 0} .
\end{gathered}
$$

The mean of (A9) is

$$
0=\left(i j \omega+\lambda_{0}\right) \mathbf{H}_{j, 0} .
$$

Thus it can be assumed

$$
\mathbf{H}_{j, 0}=0 \quad \forall j \neq 0 ; \quad \lambda_{0}=0
$$


(this is a normalisation condition: any other formal solution to (A10): $\mathbf{H}_{j, 0}=0 \forall j \neq J, \lambda_{0}=-i J \omega$ for $J \neq 0$, does not represent any new eigensolution to the original Floquet problem, since solutions $\mathbf{H}$ to the Floquet problem (4) are defined up to a factor $\left.C e^{i J \omega t}\right)$.

Consequently, the fluctuating part of (A9) yields by linearity

$$
\mathbf{G}_{j, 0}=\sum_{k=1}^{3} \mathbf{S}_{j, k}(\mathbf{x}) \mathbf{H}_{0,0}^{k}(\mathbf{y})
$$

where vector fields $\mathbf{S}_{j, k}$ satisfy

$$
\mathcal{L}_{j} \mathbf{S}_{\cdot, k}=-\frac{\partial}{\partial x_{k}}\left(\delta_{1}^{j} \mathbf{W}+\delta_{0}^{j} \mathbf{U}+\delta_{-1}^{j} \overline{\mathbf{W}}\right),
$$

which is a representation of the first auxiliary problem (19) involving notation (A2). The problem (A13) has a unique solution by the assumption that the kernel of $\mathcal{L}$ is empty. It is evident from (A13) and from the definition of the operators $\mathcal{L}_{j}$ that

$$
\mathbf{S}_{-j, k}=\overline{\mathbf{S}}_{j, k} ; \quad \nabla_{\mathbf{x}} \cdot \mathbf{S}_{j, k}=0 \quad \forall j, k .
$$

Parity invariance of the flow (2) implies that parity anti-invariant vector fields are an invariant subspace of $\mathcal{L}$, and since the right-hand side of (A13) is parity anti-invariant, so are $\mathbf{S}_{j, k}$ :

$$
\mathbf{S}_{j, k}(\mathbf{x})=\mathbf{S}_{j, k}(-\mathbf{x}) .
$$

Divergence (in fast variables) of (A13) implies that $\mathbf{S}_{j, k}$ are solenoidal.

ii. The second $(n=1)$ term of (A8) reduces with the use of (A3), (A7) and (A11) to

$$
\begin{gathered}
\mathcal{L}_{j} \mathbf{G}_{,, 1}+2 \eta\left(\nabla_{\mathbf{x}} \cdot \nabla_{\mathbf{y}}\right) \mathbf{G}_{j, 0}+\left(\mathbf{H}_{j-1,1} \cdot \nabla_{\mathbf{x}}\right) \mathbf{W}+\left(\mathbf{H}_{j, 1} \cdot \nabla_{\mathbf{x}}\right) \mathbf{U}+\left(\mathbf{H}_{j+1,1} \cdot \nabla_{\mathbf{x}}\right) \overline{\mathbf{W}} \\
+\nabla_{\mathbf{y}} \times\left(\mathbf{W} \times \mathbf{G}_{j-1,0}+\mathbf{U} \times \mathbf{G}_{j, 0}+\overline{\mathbf{W}} \times \mathbf{G}_{j+1,0}\right) \\
-\left(\left(\delta_{1}^{j} \mathbf{W}+\delta_{0}^{j} \mathbf{U}+\delta_{-1}^{j} \overline{\mathbf{W}}\right) \cdot \nabla_{\mathbf{y}}\right) \mathbf{H}_{0,0}=\lambda_{1}\left(\delta_{0}^{j} \mathbf{H}_{0,0}+\mathbf{G}_{j, 0}\right)+i j \omega \mathbf{H}_{j, 1} . \quad(\mathrm{A} 15)
\end{gathered}
$$

Upon substitution of (A12) the mean of (A15) becomes

$$
\nabla_{\mathbf{y}} \times \sum_{k=1}^{3}\left\langle\mathbf{W} \times \mathbf{S}_{j-1, k}+\mathbf{U} \times \mathbf{S}_{j, k}+\overline{\mathbf{W}} \times \mathbf{S}_{j+1, k}\right\rangle \mathbf{H}_{0,0}^{k}=\lambda_{1} \delta_{0}^{j} \mathbf{H}_{0,0}+i j \omega \mathbf{H}_{j, 1} .
$$

In view of (A4) and (A14), the averaged cross products at the left-hand side of this equation vanish and thus

$$
\mathbf{H}_{j, 1}=0 \quad \forall j \neq 0 ; \quad \lambda_{1}=0 .
$$

After (A16) and the representations (A12) are plugged in, the fluctuating part of (A15) becomes 


$$
\begin{gathered}
\mathcal{L}_{j} \mathbf{G}_{\cdot, 1}=-\left(\mathbf{H}_{0,1} \cdot \nabla_{\mathbf{x}}\right)\left(\delta_{1}^{j} \mathbf{W}+\delta_{0}^{j} \mathbf{U}+\delta_{-1}^{j} \overline{\mathbf{W}}\right) \\
+\sum_{k=1}^{3} \sum_{m=1}^{3}\left[-2 \eta \frac{\partial \mathbf{S}_{j, k}}{\partial x_{m}}+\mathbf{W}^{m}\left(\mathbf{S}_{j-1, k}+\delta_{1}^{j} \mathbf{e}_{k}\right)-\mathbf{W} \mathbf{S}_{j-1, k}^{m}\right. \\
\left.+\mathbf{U}^{m}\left(\mathbf{S}_{j, k}+\delta_{0}^{j} \mathbf{e}_{k}\right)-\mathbf{U S}_{j, k}^{m}+\overline{\mathbf{W}}^{m}\left(\mathbf{S}_{j+1, k}+\delta_{-1}^{j} \mathbf{e}_{k}\right)-\overline{\mathbf{W}} \mathbf{S}_{j+1, k}^{m}\right] \frac{\partial \mathbf{H}_{0,0}^{k}}{\partial y_{m}} .
\end{gathered}
$$

Hence by linearity

$$
\mathbf{G}_{j, 1}=\sum_{k=1}^{3} \mathbf{S}_{j, k}(\mathbf{x}) \mathbf{H}_{0,1}^{k}(\mathbf{y})+\sum_{k=1}^{3} \sum_{m=1}^{3} \boldsymbol{\Gamma}_{j, m, k}(\mathbf{x}) \frac{\partial \mathbf{H}_{0,0}^{k}}{\partial y_{m}}(\mathbf{y}),
$$

where vector fields $\boldsymbol{\Gamma}_{j, m, k}(\mathbf{x})$ satisfy

$$
\begin{gathered}
\mathcal{L}_{j} \boldsymbol{\Gamma}_{\cdot, m, k}=-2 \eta \frac{\partial \mathbf{S}_{j, k}}{\partial x_{m}}+\mathbf{W}^{m}\left(\mathbf{S}_{j-1, k}+\delta_{1}^{j} \mathbf{e}_{k}\right)-\mathbf{W} \mathbf{S}_{j-1, k}^{m} \\
+\mathbf{U}^{m}\left(\mathbf{S}_{j, k}+\delta_{0}^{j} \mathbf{e}_{k}\right)-\mathbf{U S}_{j, k}^{m}+\overline{\mathbf{W}}^{m}\left(\mathbf{S}_{j+1, k}+\delta_{-1}^{j} \mathbf{e}_{k}\right)-\overline{\mathbf{W}} \mathbf{S}_{j+1, k}^{m},
\end{gathered}
$$

which is a representation of the second auxiliary problem (20) involving notation (A2). By standard arguments it is verified that

$$
\boldsymbol{\Gamma}_{-j, m, k}=\overline{\boldsymbol{\Gamma}}_{j, m, k} ; \quad \boldsymbol{\Gamma}_{j, m, k}(\mathbf{x})=-\boldsymbol{\Gamma}_{j, m, k}(-\mathbf{x}) .
$$

Divergence (in fast variables) of (A19) implies $\nabla_{\mathbf{x}} \cdot \boldsymbol{\Gamma}_{j, m, k}+\mathbf{S}_{j, k}^{m}=0$.

iii. The third $(n=2)$ term of (A8) by virtue of (A11) and (A16) reduces to

$$
\begin{aligned}
\mathcal{L}_{j} \mathbf{G}_{\cdot, 2}+\eta\left(2\left(\nabla_{\mathbf{x}} \cdot \nabla_{\mathbf{y}}\right) \mathbf{G}_{j, 1}+\nabla_{\mathbf{y}}^{2}\left(\delta_{0}^{j} \mathbf{H}_{0,0}+\mathbf{G}_{j, 0}\right)\right) \\
+\left(\mathbf{H}_{j-1,2} \cdot \nabla_{\mathbf{x}}\right) \mathbf{W}+\left(\mathbf{H}_{j, 2} \cdot \nabla_{\mathbf{x}}\right) \mathbf{U}+\left(\mathbf{H}_{j+1,2} \cdot \nabla_{\mathbf{x}}\right) \overline{\mathbf{W}} \\
+\nabla_{\mathbf{y}} \times\left(\mathbf{W} \times \mathbf{G}_{j-1,1}+\mathbf{U} \times \mathbf{G}_{j, 1}+\overline{\mathbf{W}} \times \mathbf{G}_{j+1,1}\right) \\
-\left(\left(\delta_{1}^{j} \mathbf{W}+\delta_{0}^{j} \mathbf{U}+\delta_{-1}^{j} \overline{\mathbf{W}}\right) \cdot \nabla_{\mathbf{y}}\right) \mathbf{H}_{0,1}=\lambda_{2}\left(\delta_{0}^{j} \mathbf{H}_{0,0}+\mathbf{G}_{j, 0}\right)+i j \omega \mathbf{H}_{j, 2} .
\end{aligned}
$$

In view of (A18), (A4) and (A14) the mean of this equations is

$$
\begin{gathered}
\eta \delta_{0}^{j} \nabla_{\mathbf{y}}^{2} \mathbf{H}_{0,0}+\nabla_{\mathbf{y}} \times \sum_{m=1}^{3} \sum_{k=1}^{3}\left\langle\mathbf{W} \times \boldsymbol{\Gamma}_{j-1, m, k}+\mathbf{U} \times \boldsymbol{\Gamma}_{j, m, k}+\overline{\mathbf{W}} \times \boldsymbol{\Gamma}_{j+1, m, k}\right\rangle \frac{\partial \mathbf{H}_{0,0}^{k}}{\partial y_{m}} \\
=\lambda_{2} \delta_{0}^{j} \mathbf{H}_{0,0}+i j \omega \mathbf{H}_{j, 2} .
\end{gathered}
$$

Thus the leading terms of the expansions of the mean magnetic field, $\mathbf{H}_{0,0}$, and of the growth rate, $\lambda_{2}$, are a solution to the eigenvalue problem

$$
\begin{gathered}
\mathcal{M H}_{0,0} \equiv \eta \nabla^{2} \mathbf{H}_{0,0}+\nabla_{\mathbf{y}} \times \sum_{m=1}^{3} \sum_{k=1}^{3}\left(2 \operatorname{Re}\left\langle\overline{\mathbf{W}} \times \boldsymbol{\Gamma}_{1, m, k}\right\rangle+\left\langle\mathbf{U} \times \boldsymbol{\Gamma}_{0, m, k}\right\rangle\right) \frac{\partial \mathbf{H}_{0,0}^{k}}{\partial y_{m}} \\
=\lambda_{2} \mathbf{H}_{0,0},
\end{gathered}
$$


arising from (A21) for $j=0$. (A22) is equivalent to (9) in view of (A2). Subsequently one obtains from (A21)

$\mathbf{H}_{j, 2}=\frac{1}{i j \omega} \nabla_{\mathbf{y}} \times \sum_{m=1}^{3} \sum_{k=1}^{3}\left\langle\mathbf{W} \times \boldsymbol{\Gamma}_{j-1, m, k}+\mathbf{U} \times \boldsymbol{\Gamma}_{j, m, k}+\overline{\mathbf{W}} \times \boldsymbol{\Gamma}_{j+1, m, k}\right\rangle \frac{\partial \mathbf{H}_{0,0}^{k}}{\partial y_{m}}$

for any $j \neq 0$. Hence now the quantities $\mathbf{G}_{j, 0}$ are also entirely determined by (A12).

Bounded solutions to the eigenvalue problem (A22) are Fourier harmonics $\mathbf{H}_{0,0}=\tilde{\mathbf{h}} e^{i \mathbf{q y}}$, satisfying the orthogonality condition (14) and

$$
\eta|\mathbf{q}|^{2} \tilde{\mathbf{h}}+\mathbf{q} \times \sum_{m=1}^{3}\left(2 \operatorname{Re}\left\langle\mathbf{W} \times \boldsymbol{\Gamma}_{-1, m, k}\right\rangle+\left\langle\mathbf{U} \times \boldsymbol{\Gamma}_{0, m, k}\right\rangle\right) \mathbf{q}_{m} \tilde{\mathbf{h}}_{k}=-\lambda_{2} \tilde{\mathbf{h}},
$$

which is an equivalent of (13) in new notation (A2).

The $\mathbf{x}$-dependent prefactors in front of unknown vector fields $\mathbf{H}_{0,2}^{k}$ and $\partial \mathbf{H}_{0,1}^{k} / \partial y_{m}$ in the fluctuating part of (A20),

$$
\begin{gathered}
\mathcal{L}_{j} \mathbf{G}_{\cdot, 2}=-\eta\left(2 \sum_{k=1}^{3} \sum_{m=1}^{3}\left(\frac{\partial \mathbf{S}_{j, k}}{\partial x_{m}} \frac{\partial \mathbf{H}_{0,1}^{k}}{\partial y_{m}}+\sum_{l=1}^{3} \frac{\partial \boldsymbol{\Gamma}_{j, m, k}}{\partial x_{l}} \frac{\partial^{2} \mathbf{H}_{0,0}^{k}}{\partial y_{m} \partial y_{l}}\right)+\nabla_{\mathbf{y}}^{2} \mathbf{G}_{j, 0}\right) \\
-\left(\mathbf{H}_{j-1,2} \cdot \nabla_{\mathbf{x}}\right) \mathbf{W}-\left(\mathbf{H}_{j, 2} \cdot \nabla_{\mathbf{x}}\right) \mathbf{U}-\left(\mathbf{H}_{j+1,2} \cdot \nabla_{\mathbf{x}}\right) \overline{\mathbf{W}} \\
+\left(\left(\delta_{1}^{j} \mathbf{W}+\delta_{0}^{j} \mathbf{U}+\delta_{-1}^{j} \overline{\mathbf{W}}\right) \cdot \nabla_{\mathbf{y}}\right) \mathbf{H}_{0,1} \\
-\nabla_{\mathbf{y}} \times\left(\sum_{k=1}^{3}\left(\mathbf{W} \times \mathbf{S}_{j-1, k}+\mathbf{U} \times \mathbf{S}_{j, k}+\overline{\mathbf{W}} \times \mathbf{S}_{j+1, k}\right) \mathbf{H}_{0,1}^{k}\right. \\
\left.+\sum_{k=1}^{3} \sum_{m=1}^{3}\left\{\mathbf{W} \times \boldsymbol{\Gamma}_{j-1, m, k}+\mathbf{U} \times \boldsymbol{\Gamma}_{j, m, k}+\overline{\mathbf{W}} \times \boldsymbol{\Gamma}_{j+1, m, k}\right\} \frac{\partial \mathbf{H}_{0,0}^{k}}{\partial y_{m}}\right)+\lambda_{2} \mathbf{G}_{j, 0},
\end{gathered}
$$

are the same as those in front of $\mathbf{H}_{0,1}^{k}$ and $\partial \mathbf{H}_{0,0}^{k} / \partial y_{m}$, respectively, in (A17), which by linearity implies a representation

$$
\mathbf{G}_{j, 2}=\sum_{k=1}^{3} \mathbf{S}_{j, k}(\mathbf{x}) \mathbf{H}_{0,2}^{k}(\mathbf{y})+\sum_{k=1}^{3} \sum_{m=1}^{3} \boldsymbol{\Gamma}_{j, m, k}(\mathbf{x}) \frac{\partial \mathbf{H}_{0,1}^{k}}{\partial y_{m}}(\mathbf{y})+\mathbf{Q}_{j, 2}(\mathbf{x}, \mathbf{y}) .
$$

Vector fields $\mathbf{Q}_{j, 2}$ can be found from a system equations, obtained from (A24) by changing $\mathbf{G}_{\cdot, 2} \rightarrow \mathbf{Q}_{\cdot, 2}$ and dropping all terms involving $\mathbf{H}_{0,2}^{k}$ or derivatives of $\mathbf{H}_{0,1}^{k}$ (the right-hand sides of the resultant equations are at this stage known).

$i v$. Let $\lambda_{2}^{\prime}$ and $\mathbf{h}^{\prime}$ be the second eigenvalue and the associated eigenvector, satisfying (A23) and (14). Subsequent $(n>2)$ terms of (A8) provide a hierarchy of equations, which can be solved under the condition $\lambda_{2} \neq \lambda_{2}^{\prime}$. Equations for $n<N$ yield:

- vector fields $\mathbf{H}_{j, n}$ for all $j \neq 0$ and $n<N$;

- vector fields $\mathbf{H}_{0, n}$ for all $n<N-2$;

- vector fields $\mathbf{G}_{j, n}$ for all $j$ and $n<N-2$; 
- representations of $\mathbf{G}_{j, n}$ of the form

$$
\mathbf{G}_{j, n}=\sum_{k=1}^{3} \mathbf{S}_{j, k}(\mathbf{x}) \mathbf{H}_{0, n}^{k}(\mathbf{y})+\sum_{k=1}^{3} \sum_{m=1}^{3} \boldsymbol{\Gamma}_{j, m, k}(\mathbf{x}) \frac{\partial \mathbf{H}_{0, n-1}^{k}}{\partial y_{m}}(\mathbf{y})+\mathbf{Q}_{j, n}(\mathbf{x}, \mathbf{y})
$$

for $n=N-1$ and $n=N-2$ with known vector fields $\mathbf{Q}_{j, n},\left\langle\mathbf{Q}_{j, n}\right\rangle=0$; - quantities $\lambda_{n}$ for all $n<N$.

Upon substitution of (A25) for $n=N-1$ the mean of the equation corresponding to $n=N$ in (A8) becomes

$$
\begin{gathered}
\eta \nabla^{2} \mathbf{H}_{j, N-2}+\nabla_{\mathbf{y}} \times \sum_{m=1}^{3} \sum_{k=1}^{3}\left\langle\mathbf{W} \times \boldsymbol{\Gamma}_{j-1, m, k}+\mathbf{U} \times \boldsymbol{\Gamma}_{j, m, k}+\overline{\mathbf{W}} \times \boldsymbol{\Gamma}_{j+1, m, k}\right\rangle \frac{\partial \mathbf{H}_{0, N-2}^{k}}{\partial y_{m}} \\
+\nabla_{\mathbf{y}} \times\left\langle\mathbf{W} \times \mathbf{Q}_{j-1, N-1}+\mathbf{U} \times \mathbf{Q}_{j, N-1}+\overline{\mathbf{W}} \times \mathbf{Q}_{j+1, N-1}\right\rangle \\
=\sum_{m=0}^{N-2} \lambda_{N-m} \mathbf{H}_{j, m}+i j \omega \mathbf{H}_{j, N} .
\end{gathered}
$$

Consider (A26) for $j=0$ :

$$
\begin{gathered}
\left(\mathcal{M}-\lambda_{2}\right) \mathbf{H}_{0, N-2}-\lambda_{N} \mathbf{H}_{0,0} \\
=\sum_{m=1}^{N-3} \lambda_{N-m} \mathbf{H}_{0, m}-\nabla_{\mathbf{y}} \times\left(2 \operatorname{Re}\left\langle\overline{\mathbf{W}} \times \mathbf{Q}_{1, N-1}\right\rangle+\left\langle\mathbf{U} \times \mathbf{Q}_{0, N-1}\right\rangle\right),
\end{gathered}
$$

where the right-hand side is a known vector field. Projecting this equation out in the direction of $\mathbf{H}_{0,0}$ one can uniquely determine $\lambda_{N}$. In the complementary invariant subspace of $\mathcal{M}$ the operator $\mathcal{M}-\lambda_{2}$ is invertible, and thus $\mathbf{H}_{0, N-2}$ can be determined from (A27) up to an arbitrary multiple of $\mathbf{H}_{0,0}$, which we can demand to vanish. Now $\mathbf{H}_{j, N}$ for $j \neq 0$ can be found from (A26), and $\mathbf{G}_{j, N-2}$ are determined by (A25) for $n=N-2$.

The fluctuating part of the equation corresponding to $n=N$ in (A8) becomes after the substitution of (A25) for $n=N-1$

$$
\begin{aligned}
& \mathcal{L}_{j} \mathbf{G}_{\cdot, N}=-\eta\left(2 \sum_{k=1}^{3} \sum_{m=1}^{3}\left(\frac{\partial \mathbf{S}_{j, k}}{\partial x_{m}} \frac{\partial \mathbf{H}_{0, N-1}^{k}}{\partial y_{m}}+\sum_{l=1}^{3} \frac{\partial \boldsymbol{\Gamma}_{j, m, k}}{\partial x_{l}} \frac{\partial^{2} \mathbf{H}_{0, N-2}^{k}}{\partial y_{l} \partial y_{m}}\right)\right. \\
& \left.+2\left(\nabla_{\mathbf{x}} \cdot \nabla_{\mathbf{y}}\right) \mathbf{Q}_{j, N-1}+\nabla_{\mathbf{y}}^{2} \mathbf{G}_{j, N-2}\right)-\left(\mathbf{H}_{j-1, N} \cdot \nabla_{\mathbf{x}}\right) \mathbf{W}+\left(\mathbf{W} \cdot \nabla_{\mathbf{y}}\right) \mathbf{H}_{j-1, N-1} \\
& -\left(\mathbf{H}_{j, N} \cdot \nabla_{\mathbf{x}}\right) \mathbf{U}+\left(\mathbf{U} \cdot \nabla_{\mathbf{y}}\right) \mathbf{H}_{j, N-1}-\left(\mathbf{H}_{j+1, N} \cdot \nabla_{\mathbf{x}}\right) \overline{\mathbf{W}}+\left(\overline{\mathbf{W}} \cdot \nabla_{\mathbf{y}}\right) \mathbf{H}_{j+1, N-1} \\
& -\nabla_{\mathbf{y}} \times\left[\sum_{k=1}^{3}\left(\mathbf{W} \times \mathbf{S}_{j-1, k}+\mathbf{U} \times \mathbf{S}_{j, k}+\overline{\mathbf{W}} \times \mathbf{S}_{j+1, k}\right) \mathbf{H}_{0, N-1}^{k}\right. \\
& +\sum_{k=1}^{3} \sum_{m=1}^{3}\left\{\mathbf{W} \times \boldsymbol{\Gamma}_{j-1, m, k}+\mathbf{U} \times \boldsymbol{\Gamma}_{j, m, k}+\overline{\mathbf{W}} \times \boldsymbol{\Gamma}_{j+1, m, k}\right\} \frac{\partial \mathbf{H}_{0, N-2}^{k}}{\partial y_{m}} \\
& \left.\left.+\left\{\mathbf{W} \times \mathbf{Q}_{j-1, N-1}+\mathbf{U} \times \mathbf{Q}_{j, N-1}+\overline{\mathbf{W}} \times \mathbf{Q}_{j+1, N-1}\right)\right\}\right]+\sum_{m=0}^{N-2} \lambda_{N-m} \mathbf{G}_{j, m} .
\end{aligned}
$$


Like in the case of (A24), the structure of this equation implies by linearity the representation (A25) of $\mathbf{G}_{j, N}$, where the quantities $\mathbf{Q}_{j, N}$ are uniquely defined by

$$
\begin{aligned}
& \mathcal{L}_{j} \mathbf{Q} \cdot N=-\eta\left(2 \sum_{k=1}^{3} \sum_{m=1}^{3} \sum_{l=1}^{3} \frac{\partial \boldsymbol{\Gamma}_{j, m, k}}{\partial x_{l}} \frac{\partial^{2} \mathbf{H}_{0, N-2}^{k}}{\partial y_{l} \partial y_{m}}+2\left(\nabla_{\mathbf{x}} \cdot \nabla_{\mathbf{y}}\right) \mathbf{Q}_{j, N-1}+\nabla_{\mathbf{y}}^{2} \mathbf{G}_{j, N-2}\right) \\
& -\left(1-\delta_{1}^{j}\right)\left(\mathbf{H}_{j-1, N} \cdot \nabla_{\mathbf{x}}\right) \mathbf{W}-\left(1-\delta_{0}^{j}\right)\left(\mathbf{H}_{j, N} \cdot \nabla_{\mathbf{x}}\right) \mathbf{U}-\left(1-\delta_{-1}^{j}\right)\left(\mathbf{H}_{j+1, N} \cdot \nabla_{\mathbf{x}}\right) \overline{\mathbf{W}} \\
& +\left(1-\delta_{1}^{j}\right)\left(\mathbf{W} \cdot \nabla_{\mathbf{y}}\right) \mathbf{H}_{j-1, N-1}+\left(1-\delta_{1}^{j}\right)\left(\mathbf{U} \cdot \nabla_{\mathbf{y}}\right) \mathbf{H}_{j, N-1}+\left(1-\delta_{-1}^{j}\right)\left(\overline{\mathbf{W}} \cdot \nabla_{\mathbf{y}}\right) \mathbf{H}_{j+1, N-1} \\
& -\nabla_{\mathbf{y}} \times\left[\sum_{k=1}^{3} \sum_{m=1}^{3}\left\{\mathbf{W} \times \boldsymbol{\Gamma}_{j-1, m, k}+\mathbf{U} \times \boldsymbol{\Gamma}_{j, m, k}+\overline{\mathbf{W}} \times \boldsymbol{\Gamma}_{j+1, m, k}\right\} \frac{\partial \mathbf{H}_{0, N-2}^{k}}{\partial y_{m}}\right. \\
& \left.\left.\quad+\left\{\mathbf{W} \times \mathbf{Q}_{j-1, N-1}+\mathbf{U} \times \mathbf{Q}_{j, N-1}+\overline{\mathbf{W}} \times \mathbf{Q}_{j+1, N-1}\right)\right\}\right]+\sum_{m=0}^{N-2} \lambda_{N-m} \mathbf{G}_{j, m}
\end{aligned}
$$

(the right-hand side of this equation is known). This equation was obtained by omitting in (A28) all terms, involving $\mathbf{H}_{0, N}$ or derivatives of $\mathbf{H}_{0, N-1}$, and changing $\mathbf{G}_{\cdot, N}$ to $\mathbf{Q}_{\cdot, N}$.

Thus a complete asymptotic expansion of magnetic modes and their growth rates is constructed. Like in the case of a stationary velocity, it can be easily verified that

$$
\mathbf{H}_{0,0}=\mathbf{h} e^{i \mathbf{q y}}, \quad \mathbf{H}_{0, n}=\chi_{n} \mathbf{h}^{\prime} e^{i \mathbf{q y}} \quad \forall n>0, \quad \mathbf{H}_{j, n}=\mathbf{h}_{j, n} e^{i \mathbf{q y}} \quad \forall n, j \neq 0
$$

(where $\mathbf{h}_{j, n}=$ const, $\mathbf{h}_{j, n} \cdot \mathbf{q}=0$ ),

$$
\mathbf{G}_{j, n}=\mathbf{g}_{n}(\mathbf{x}) e^{i \mathbf{q y}} \quad \forall n \geq 0
$$

and thus the eigenmode admits a representation

$$
\mathbf{H}=e^{i \epsilon \mathbf{q} \mathbf{x}} \mathbf{h}(\mathbf{x}, t), \quad \mathbf{q}=\text { const. }
$$

(For this reason for constructions of section $i v$ it was sufficient to demand that $\lambda^{\prime} \neq \lambda_{2}$, and not that $\mathcal{M}-\lambda_{2}$ is invertible in the whole domain.) This stems from the fact that for the velocity (A1) the domain of the magnetic induction operator splits into invariant subspaces, each comprised of vector fields (A29) and categorised by wavevectors $\mathbf{q}$.

Analyzing parity of solutions of the hierarchy of equations (A8) constructed in this Appendix, one finds that all $\mathbf{G}_{j, n}$ with even indices $n$ are parity anti-invariant; all $\mathbf{G}_{j, n}$ with odd indices $n$ are parity-invariant; $\left\langle\mathbf{H}_{j, n}\right\rangle=\mathbf{0}$ for any odd $n$; and $\lambda_{n}=0$ for any odd $n$. 